\title{
Measurement of Fracture Parameters for a Mixed-Mode Crack Driven by Stress Waves using Image Correlation Technique and High-Speed Digital Photography
}

\author{
M. S. Kirugulige* and H. V. Tippur ${ }^{\dagger}$ \\ *The Goodyear Tire and Rubber Co., 142 Goodyear Blvd, Akron, OH 44305, USA \\ †Department of Mechanical Engineering, Auburn University, Auburn, AL 36849, USA
}

\begin{abstract}
Measurement of fracture parameters for a rapidly growing crack in syntactic foam sheets using image correlation technique and high-speed photography is presented. The performance of a rotating mirror-type multi-channel high-speed digital camera to measure transient deformations is assessed by conducting benchmark tests on image intensity variability, rigid translation and rigid rotation. Edge-cracked foam samples are subjected to eccentric impact loading relative to the initial crack plane to produce mixed-mode loading conditions in a three-point bend configuration. High-speed photography is used to record decorated random speckles in the vicinity of the crack tip at a rate of 200000 frames per second. Two sets of images are recorded, the first set before impact and the second after impact. Using image correlation methodology, crack-tip displacement field histories and dominant strains from the time of impact up to complete fracture are mapped. Over-deterministic least-squares analyses of crack-tip radial and tangential displacements are used to obtain mixed-mode fracture parameters. The measurements are compared with complementary finite element results. The fracture parameters determined from radial displacements seem more robust even when fewer number of higher order terms in the crack-tip asymptotic expansion are used.
\end{abstract}

KEY WORDS: DIC, dynamic crack growth, high-speed imaging, mixed-mode SIF, optical metrology, stress wave loading

\section{Introduction}

The mixed-mode dynamic fracture behaviour of syntactic foams is examined in this study. Syntactic foams are lightweight structural materials manufactured by dispersing prefabricated hollow microballoons in a matrix. These materials also display superior thermal, dielectric, fire-resistant, hygroscopic properties and sometimes radar or sonar transparency. They can also be tailored to suit a particular application by selecting microballoons made of glass, carbon or polymer to be used with different matrix materials (metal, polymer or ceramic). Although syntactic foams were initially developed for deep-sea applications, in recent years they have found a variety of applications such as buoyancy modules for boat hulls, parts of helicopters and airplanes, structural components of antenna assemblies, thermal insulators in oil and gas industries, core materials in impact-resistant sandwich structures, to name a few. The ability to absorb impact energy and vibrations makes them ideal candidates for packaging applications and protective enclosures. Although more suitable for compression-dominated applications, structural components made of syntactic foams often undergo a combined loading including shock, resulting in tensile or shear failures. Hence studying the mixed-mode fracture response under stress wave-dominant conditions is critical for this material.

Choices in terms of experimental techniques for measuring real-time surface deformations/stresses in a dynamic failure event such as fracture initiation and propagation are somewhat limited. Dynamic photoelasticity [1-3], coherent gradient sensing [4-7] and moiré interferometry [8] have emerged over the years as full-field techniques suitable for investigating highly transient events such as crack initiation and propagation in solids. Interferometric techniques, however, involve elaborate surface preparation [transferring of gratings in case of moiré interferometry and preparing a specularly reflective surface in 
case of coherent gradient sensing (CGS), birefringent coatings in reflection photoelasticity, etc.]. For cellular materials (syntactic foams, polymer metal foams, cellulosic materials, etc.) such surface preparations are challenging and in some cases may not be feasible. In those instances, digital image correlation could be a useful tool because of its relative simplicity. It involves a rather simple surface preparation method of decorating the surface with random speckles using alternate mists of black and white paint. Recent advances in image processing and ubiquitous computational capabilities have made it possible to apply this technique to a variety of engineering applications including mixed-mode crack growth studies under static loading conditions $[9,10]$.

With the advent of high-speed digital cameras in recent years, recording rates as high as several million frames per second at a relatively high spatial resolution have become possible. This has made image correlation techniques feasible for estimating surface displacements and strains to assess fracture/damage parameters. In this study, the digital image correlation technique is extended for the first time to mixed-mode dynamic fracture studies for estimating stress intensity factors (SIF) of stationary and propagating cracks in edge-cracked syntactic foam sheets subjected to stress wave loading. A rotating mirrortype high-speed digital camera is used to record random speckles in the vicinity of the crack tip. The entire crack-tip deformation and dominant strain history from the time of impact to complete fracture is mapped. Over-deterministic least-squares analyses of crack-tip displacement fields are performed to obtain dynamic SIF histories for both pre- and postcrack initiation periods. The SIF histories obtained from the image correlation method are compared with those obtained from finite element computations.

\section{The Approach}

In this study, random speckle patterns on the specimen surface were monitored during a dynamic mixed-mode fracture event. Two sets of these patterns, the first set before and the second set after deformation, were acquired, digitised and stored. Then a sub-image in an undeformed image was chosen and its location in the corresponding deformed image was sought (see Figure 1). Once the location was identified, the local displacements were quantified. In this study, a three-step approach was used in a MATLAB ${ }^{\mathrm{TM}}$ [11] environment to estimate in-plane surface displacements and strains. In the

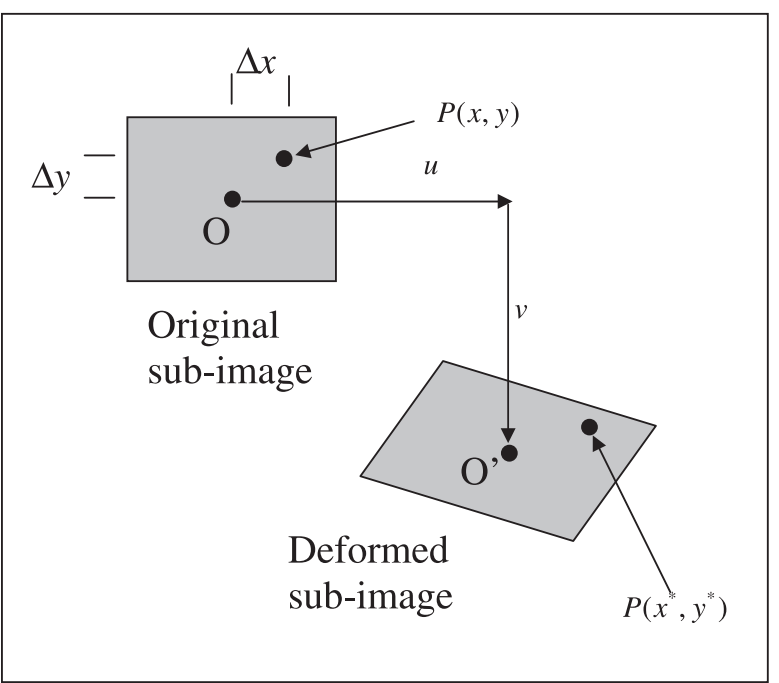

Figure I: Undeformed and deformed sub-images chosen from images before and after deformation respectively

following only a brief description of all the steps involved is provided, and details can be found in Ref. $[12,13]$.

In the first step, a 2D cross-correlation coefficient was computed to obtain initial estimates of full-field planar displacements. The peak of the correlation function was detected to sub-pixel accuracy (1/16th of a pixel) using bicubic interpolation. This process was repeated for the entire image to get full-field in-plane displacements. Further details about this method can be found in Ref. [14]. In the second step, an iterative technique based on nonlinear leastsquares minimisation was implemented to estimate displacements and their gradients by using displacements obtained in the first step as initial guess values. The Newton-Raphson method, which uses line search and the BFGS (Broyden, Fletcher, Goldfarb and Shanno) algorithm to update an inverse Hessian matrix, was employed. Such an approach was demonstrated first by Sutton et al. [15] to measure displacements from speckle images. The displacement gradients obtained during the second step represent average values for each subset and tend to be noisy. Therefore, it was necessary to use smoothing algorithms in order to get a continuous displacement field $(u, v)$ and then to extract strain values. It should be noted here that crack-opening displacements are discontinuous across the crack. Generic smoothing methods smooth displacements across the crack faces and hence interpretation of deformations near the crack tip tends to be inaccurate. Therefore a smoothing method which allows discontinuity of crack-opening displacements across the crack faces was introduced. A regularised restoration filter [16] with a second-order fit was employed for this 
purpose. Details are again avoided here for brevity and can be found in Ref. [12].

\section{Experimental Setup}

A schematic of the experimental setup used in this study is shown in Figure 2. The setup included an Instron-Dynatup 9250-HV (Instron, Norwood, MA, USA) drop tower for impact loading the specimen and a Cordin 550 ultrahigh-speed digital camera (Cordin Scientific Imaging, Salt Lake City, UT, USA) for recording images in real time. The drop tower had an instrumented tup for recording the impact force history and a pair of anvils for recording support reaction histories separately. The setup also had a delay/pulse generator to produce a trigger pulse when the impactor tup contacted the specimen. As all images were recorded during the dynamic event, lasting over a hundred microseconds, the setup used two high-energy flash lamps, triggered by the camera, to illuminate the specimen. The setup also utilised two computers, one to record the tup force and anvil reaction histories (5 $\mathrm{MHz}$ acquisition rate) and the other to record the images.

\section{Camera Performance Evaluation}

High-speed digital recording devices are broadly classified into two types based on the sensors they use. They are Complementary Metal Oxide Semiconductor (CMOS) and Charged Coupled Device (CCD) sensor-based digital cameras. The former can record images at moderate rates (typically less than 10000 frames per second at full resolution), whereas the latter can reach rates of 100 million frames per second. These ultrahigh-speed CCD cameras contain multiple sensors triggered electronically to achieve higher framing rates. They can be further classified into two types based on how the individual CCD sensors receive light from an objective lens. The first type uses stationary optical elements (beam splitters, lenses, etc.) with photomultiplier tubes (PMT) to amplify the light signal. In the second, however, a high-speed rotating mirror is used to distribute light to individual sensors by sweeping the image over them. It should be noted here that both the above types of CCD cameras introduce geometrical distortions/misalignments between successive images. The accuracy and repeatability of displacements measured from these using the image correlation approach outlined earlier are directly affected by optical misalignments. In the intensified CCD-type camera with PMT devices, image distortions arise because of two reasons: (i) different optical paths in the image formation process and (ii) random noise involved in multiplying photons by image intensifiers and fibre optic bundles. In the rotating mirror-type camera system, the distortions are limited to different optical paths for individual CCD sensors. In view of this, and despite relatively lower recording rates compared with the former type, good accuracy and repeatability are possible. Details about distortions in high-speed cameras and their corrections as related to image correlation are discussed in Ref. [17].

The high-speed camera system adopted in this study uses a combination of CCD-based imaging technology and high-speed rotating mirror optical system. It is capable of imaging rates of up to 2 million frames per second at a resolution of $1000 \times 1000$ pixels per image. It has 32 independent CCD image sensors positioned radially around a rotating mirror which sweeps light over these sensors (Figure 3). Each

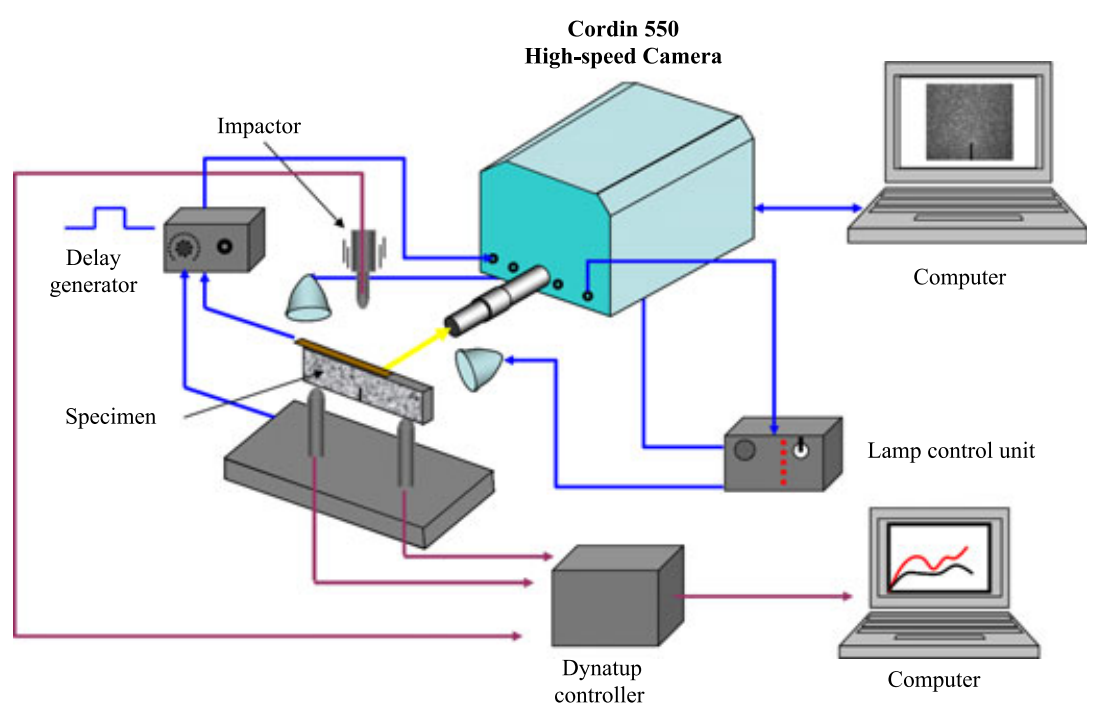

Figure 2: Schematic of the dynamic experimental setup 


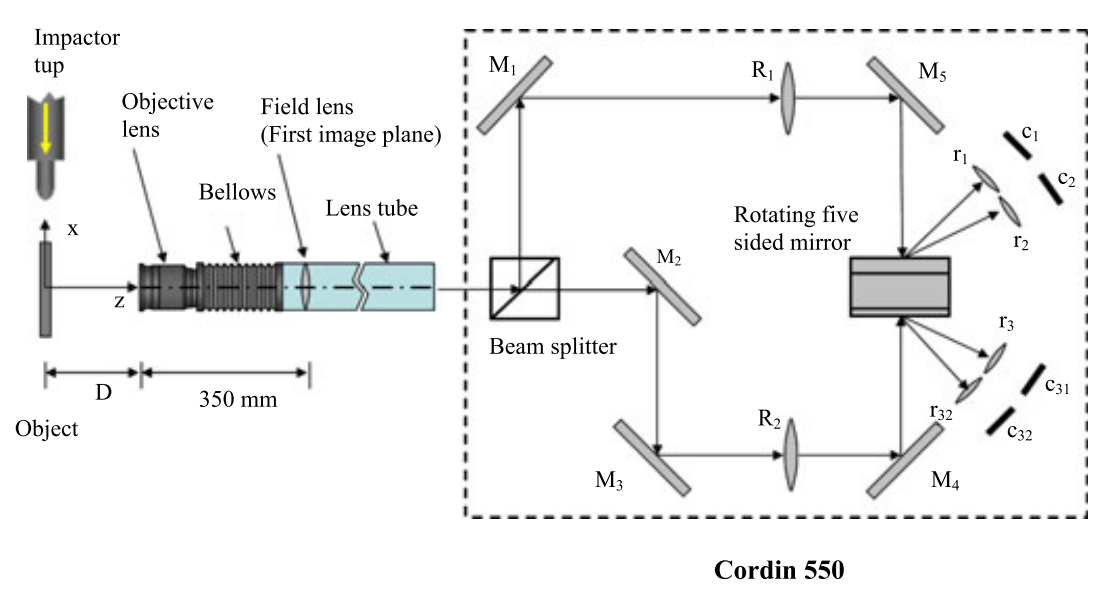

Figure 3: Optical schematic of cordin-550 camera: M1, M2, M3, M4, M5 are mirrors; R1 and R2 are relay lenses; r1, r2, .., r32 are relay lenses for CCDs; $1, \mathrm{c} 2, \ldots, \mathrm{c} 32$ are CCD sensors

sensor is illuminated by a separate optical relay. Thus small misalignments and light intensity and optical focus variations between images are unavoidable. Hence, meaningful results pertaining to small deformations cannot be obtained by correlating images from two successive/different CCD sensors. However, the above artefacts are negligible, if not entirely absent, between two images if they are captured by a same CCD sensor at two different time instants. This enables digital image correlation method for quantifying surface deformations. Accordingly, the following approach was adopted in this study.

Prior to impact-loading the specimen, a set of 32 images of surface speckles were recorded at the desired framing rate $(200,000$ frames per second in this study). While keeping all the camera settings (CCD gain, flash lamp duration, framing rate, trigger delay, etc.) same, the next set of images, this time triggered by the impact event, was captured. Thereby each image in the deformed set had a corresponding image in the undeformed set. That is, if an image in the deformed set was recorded for example by sensor no. 10, then the image recorded by the same sensor (no. 10) in the undeformed set was chosen for performing image correlation operations. By adopting this method, the optical path was maintained the same for the two images under consideration and the only source of error now was the CCD noise (in the range of 4 to 6 grey levels in an 8-bit (256 grey levels) intensity image to be discussed next). It is worth noting that in order to get meaningful results it is essential that extraneous camera movements do not occur while recording a set of images and during the time interval between the two sets of images. This was achieved rather easily by triggering the camera electronically and anchoring the camera mechanically.

In view of the above-mentioned distortions/misalignments, it was important to assess the camera performance to measure transient deformations in a dynamic test. Therefore a few benchmark tests image intensity variability test, translation and rotation tests - were first conducted.

\section{Image intensity variability test}

In this study, 8-bit ( 0 to 255 levels) grey-scale images were captured and analysed to estimate CCD noise levels. The noise in an acquired image was dependent on the value of CCD gain that can be pre-set on a $0-1000$ scale. For all the experiments reported in this paper, the gain was set in the range of 500 to 550 . A value of more than 700 was the upper limit as it resulted in saturation of a few pixels in the acquired images and hence was avoided. For evaluation purposes, two sets of 32 images were acquired at framing rates of 200000 and 50000 frames per second in total darkness (with the lens cover to prevent light transmission into the camera cavity). All the images in these two sets had their pixels representing the grey-scale values in the range 0 to 8 . Hence, the lower 3 bits in an 8-bit image represents CCD noise and the intensity represented by the remaining 5 bits can be faithfully measured. Figure 4 shows mean and standard deviations of intensity values of all pixels (1 million pixels in a $1000 \times 1000$ pixel image) of the images captured in darkness. It can be seen from this figure that all the images have their mean intensity values in the range 6 to 8 with a very narrow spread (standard deviation in the range 2 to 4 ).

As mentioned earlier, in the current study, transient deformations were estimated by performing image correlation between two images acquired from the same CCD sensor, one before and another after impact. Therefore it is important to know intensity variations between the two images taken by the same CCD sensor at different instants of time. To this end, five sets of 32 images of a stationary sample, 


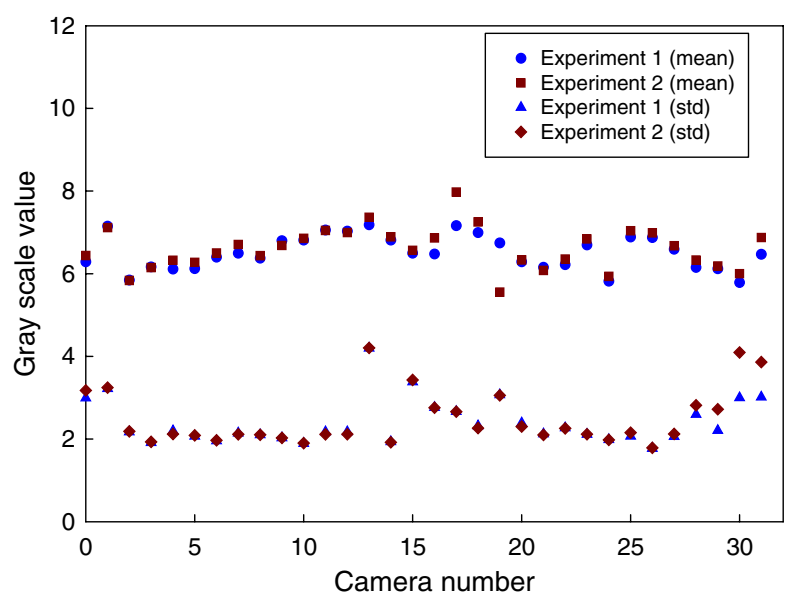

Figure 4: Mean and standard deviations of intensity values of images recorded in total darkness. Images were recorded at 50000 frames per second in experiment 1 and at 200000 frames per second in experiment 2

decorated with a random speckle pattern, were acquired at 200000 frames per second. The grey-scale values at a few randomly chosen pixels were stored (same set of pixels were chosen from all the images). The intensity value at a particular pixel from all the five images acquired by the same CCD sensor was examined. This is listed in Table 1 for all the 32 CCD sensors. As expected, a significant difference in intensity value at a pixel exists between images acquired by different CCD sensors. More importantly, however, a very small variation exists in the greyscale value at that pixel for images acquired by the same CCD sensor. The standard deviations are in the range of 2 to 6 grey levels for most sensors (apparently this is in the same range as the mean values observed for the images recorded in total darkness; see Figure 4). This demonstrates that between an undeformed and a deformed image recorded during an actual experiment, there would be no light intensity variation apart from random CCD noise. This is a subtle point but an important aspect of the high-speed camera system used here which makes it possible to perform image correlation between two

\begin{tabular}{|c|c|c|c|c|c|c|c|}
\hline Camera no. & Set I & Set 2 & Set 3 & Set 4 & Set 5 & Mean & SD \\
\hline 0 & $12 \mid$ & 116 & 123 & 120 & 122 & 120.4 & 2.70 \\
\hline I & 106 & 102 & 104 & 108 & 102 & 104.4 & 2.61 \\
\hline 2 & 119 & 120 & 125 & 116 & 120 & 120 & 3.24 \\
\hline 3 & 93 & 95 & 111 & 109 & 102 & 102 & 8.06 \\
\hline 4 & 122 & 125 & 126 & 116 & 120 & 121.8 & 4.02 \\
\hline 5 & 97 & 102 & 106 & 105 & 105 & 103 & 3.67 \\
\hline 6 & 106 & 97 & 108 & 112 & 103 & 105.2 & 5.63 \\
\hline 7 & 79 & 80 & 74 & 82 & 74 & 77.8 & 3.63 \\
\hline 8 & 84 & 81 & 84 & 89 & 84 & 84.4 & 2.88 \\
\hline 9 & 123 & 118 & 128 & 129 & 129 & 125.4 & 4.83 \\
\hline 10 & III & 105 & 110 & 114 & 116 & 111.2 & 4.21 \\
\hline II & 118 & 112 & 111 & 110 & 117 & 113.6 & 3.65 \\
\hline 12 & 82 & 88 & 76 & 82 & 82 & 82 & 4.24 \\
\hline 13 & 117 & 115 & 115 & 114 & 117 & 115.6 & 1.34 \\
\hline 14 & 88 & 98 & 93 & 87 & 93 & 91.8 & 4.44 \\
\hline 15 & 94 & 96 & 93 & 92 & 91 & 93.2 & 1.92 \\
\hline 16 & 77 & 73 & 73 & 77 & 72 & 74.4 & 2.41 \\
\hline 17 & 63 & 60 & 59 & 65 & 63 & 62 & 2.45 \\
\hline 18 & 97 & 93 & 92 & 93 & 98 & 94.6 & 2.70 \\
\hline 19 & 76 & 66 & 71 & 72 & 72 & 71.4 & 3.58 \\
\hline 20 & 69 & 70 & 60 & 71 & 67 & 67.4 & 4.39 \\
\hline 21 & 87 & 86 & 93 & 95 & 85 & 89.2 & 4.49 \\
\hline 22 & 114 & 109 & 113 & 115 & 110 & 112.2 & 2.59 \\
\hline 23 & 82 & 80 & 79 & 82 & 76 & 79.8 & 2.49 \\
\hline 24 & 92 & 89 & 93 & 96 & 84 & 90.8 & 4.55 \\
\hline 25 & 124 & 119 & 120 & 130 & 122 & 123 & 4.36 \\
\hline 26 & 122 & 120 & 126 & 117 & 123 & 121.6 & 3.36 \\
\hline 27 & 78 & 83 & 86 & 79 & 78 & 80.8 & 3.56 \\
\hline 28 & 76 & 74 & 73 & 77 & 70 & 74 & 2.74 \\
\hline 29 & 95 & 93 & 92 & 91 & 96 & 93.4 & 2.07 \\
\hline 30 & 73 & 71 & 70 & 71 & 69 & 70.8 & 1.48 \\
\hline 31 & 95 & 91 & 95 & 96 & 88 & 93 & 3.39 \\
\hline
\end{tabular}

Table I: Grey-scale values at a particular pixel in five repeated sets of images of speckle pattern acquired at 200000 frames per second

Note the consistency in grey-scale values of a camera no. (each row).

Between different camera numbers, however, grey-scale variations are anticipated. 


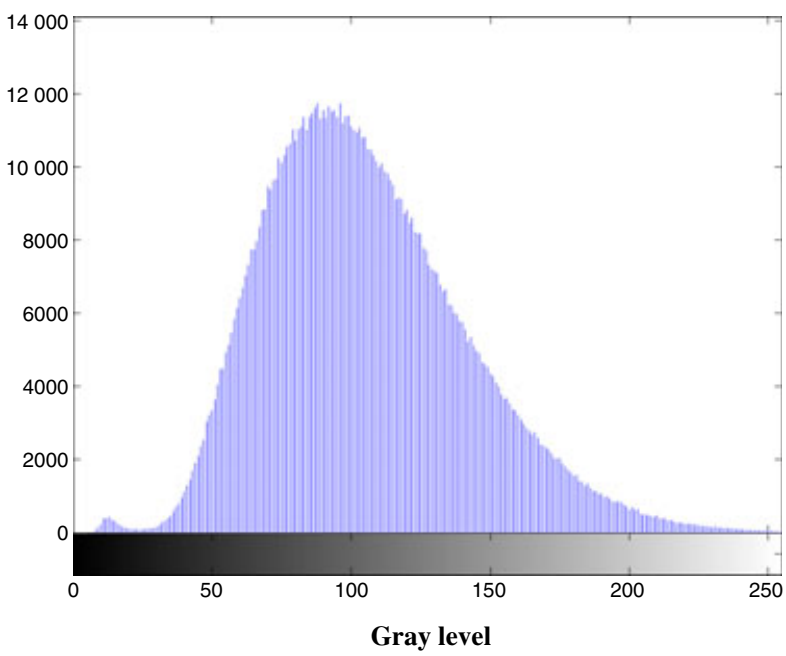

Figure 5: Histogram depicting pixel-level intensities (grey levels) of a decorated black/white speckle image acquired by a $1000 \times 1000 \mathrm{CCD}$ sensor. The vertical axis denotes the number of pixels

images acquired from the same CCD sensor and estimate displacements.

In Figure 5, a typical histogram of an image (camera no. 09) is shown to demonstrate the quality of speckles based on the CCD gain settings used during experiments. As discussed earlier in this section, lighting and CCD gains were so adjusted that the intensity levels were in the mid-range of grey scales between 0 and 255. An approximately normal distribution of intensity in the histogram plot shows the quality of random speckles in these images. (The smaller peak in the histogram near zero grey level is due to the crack where the pixels were relatively dark.)

\section{Translation test}

In these experiments, a specimen [decorated with a random black/white (b/w) speckle pattern] was mounted on a 3D-translation stage. A series of known displacements were imposed in the $X$ - and $Y$-directions separately and images captured. The mean and standard deviations of the displacement fields were computed and compared with the applied values. Moreover, an out-of-plane (Z-direction) displacement of $30 \mu \mathrm{m}^{1}$ was applied to the sample and a set of images were captured. Specific details about this test as well as the discussion of the results are reported in

\footnotetext{
${ }^{1}$ This is typically the amount of out-of-plane displacement that occurs in the vicinity of a crack tip in an experiment conducted in this work. For example, in Ref. [14] one can see roughly 7-9 fringes near the crack tip over a distance of $\sim 10 \mathrm{~mm}$. Since these fringes represent surface slopes and the resolution of the CGS set-up is $\sim 0.015^{\circ} /$ fringe, one can estimate the out-of-plane displacement around the crack tip to be $\sim 23 \mu \mathrm{m}$.
}

Ref. [12] and are avoided here for brevity. The results obtained experimentally match very well with the imposed values.

It is instructive to study in-plane strain fields estimated from the measured displacements in these translation tests. To this end, the displacement field was smoothed by the restoration method [12] and strains were obtained by numerical differentiation. The mean and standard deviations of $\varepsilon_{x x}$ and $\varepsilon_{y y}$ strains thus obtained are presented for the two tests in Table 2. These tests correspond to the $X$ - and $Y$ translations of $60 \pm 2$ and $300 \pm 2 \mu \mathrm{m}$. The applied displacement being a rigid translation, ideally zero strains are expected for all the images. However, numerical differentiation of noisy displacement data produces non-zero strains. The mean values of strains thus obtained are in the range of approximately 0 to $\pm 300 \mu \varepsilon$ in both the experiments. The standard deviations of strains are in the range 0 to $300 \mu \varepsilon$ for various individual cameras. Interestingly, the mean and standard deviations remain unaffected by the amount of imposed translation. The implication of these in an actual experiment is that a relatively large rigid-body motion can be accommodated without sacrificing accuracy in the measured displacements and strains.

\section{Rotation test}

The objectives of the rotation test were: (a) to estimate the accuracy with which a pure rotation can be measured using this camera system; (b) to compare the performance of different individual cameras when they were used to measure the same applied rotation; and (c) to examine whether the applied rigid rotation produces any spurious strains. In the rotation test, a specimen decorated with random $\mathrm{b} / \mathrm{w}$ speckle pattern was mounted on a rotation stage. Two sets of 32 images were recorded at 200000 frames per second, one set before and another after imposing a rotation of $0.32 \pm 0.02^{\circ}$. The full-field displacements between these two sets of images were computed. The sub-image size used in the analysis was $30 \times 30$ pixels so that displacements were available after computations on a $32 \times 32(=1024)$ grid of points. These displacements were smoothed by the restoration method explained in Ref. [12]. The cross derivatives $\partial u / \partial Y$ and $\partial v / \partial X$ were computed by numerical differentiation of displacement components and rotation $\omega_{X Y}$ was then computed as,

$\omega_{X Y}=\frac{1}{2}\left(\frac{\partial u}{\partial Y}-\frac{\partial v}{\partial X}\right)$

Figure 6 shows a plot of full-field $\omega_{X Y}$ from a pair of images. The estimated values are close to the applied 


\begin{tabular}{|c|c|c|c|c|c|c|c|c|}
\hline \multirow[b]{3}{*}{ Camera no. } & \multicolumn{4}{|c|}{$\begin{array}{l}X_{\text {trans }}=60 \pm 2 \mu \mathrm{m}, Y_{\text {trans }}= \\
60 \pm 2 \mu \mathrm{m}\end{array}$} & \multicolumn{4}{|c|}{$\begin{array}{l}X_{\text {trans }}=300 \pm 2 \mu \mathrm{m}, Y_{\text {trans }}= \\
300 \pm 2 \mu \mathrm{m}\end{array}$} \\
\hline & \multicolumn{2}{|c|}{$\varepsilon_{x x}(\mu \varepsilon)$} & \multicolumn{2}{|c|}{$\varepsilon_{y y}(\mu \varepsilon)$} & \multicolumn{2}{|l|}{$\varepsilon_{x x}(\mu \varepsilon)$} & \multicolumn{2}{|l|}{$\varepsilon_{y y}(\mu \varepsilon)$} \\
\hline & Mean & SD & Mean & SD & Mean & SD & Mean & SD \\
\hline 0 & -21 & 203 & 260 & 217 & I & 209 & 2 & 229 \\
\hline I & -65 & 89 & 191 & 259 & -35 & $24 I$ & 15 & 245 \\
\hline 2 & -200 & 93 & 125 & 200 & 1 & 249 & 79 & 254 \\
\hline 3 & -210 & 140 & 30 & 209 & 14 & $34 I$ & 106 & 231 \\
\hline 4 & -119 & 70 & 154 & 185 & 2 & 250 & -10 & 241 \\
\hline 5 & -84 & 103 & 54 & 102 & -52 & 168 & 11 & 336 \\
\hline 6 & -68 & 147 & 68 & 125 & -179 & 159 & 52 & 254 \\
\hline 7 & -130 & 186 & 27 & 134 & -66 & 196 & 85 & 278 \\
\hline 8 & 0 & 95 & 100 & 157 & -20 & 211 & 79 & 196 \\
\hline 9 & -44 & 118 & 72 & 154 & 11 & 220 & 117 & 206 \\
\hline 10 & -28 & 112 & 107 & 159 & -1 & 215 & 45 & 237 \\
\hline 11 & -2 & 121 & 52 & 204 & -2 & 219 & 84 & 214 \\
\hline 12 & -15 & 128 & 125 & 203 & -42 & 201 & -5 & 215 \\
\hline 13 & -8 & $|4|$ & 37 & 285 & -14 & 193 & 79 & 196 \\
\hline 14 & 11 & 135 & 73 & 211 & -51 & 236 & 4 & 202 \\
\hline 15 & 11 & 85 & 15 & 315 & -17 & 217 & 102 & 59 \\
\hline 16 & -7 & 103 & 169 & 308 & -76 & 247 & 51 & 92 \\
\hline 17 & -15 & 113 & 4 & 319 & -8 & 239 & 17 & 169 \\
\hline 18 & 56 & 165 & 60 & 259 & 7 & 222 & 88 & 244 \\
\hline 19 & -8 & 126 & 192 & 215 & -3 & 242 & -33 & 243 \\
\hline 20 & 32 & 117 & 89 & 242 & -70 & 215 & 22 & 249 \\
\hline 21 & 15 & 105 & 189 & 148 & -17 & 288 & 55 & 235 \\
\hline 22 & 28 & 150 & 196 & 167 & -40 & 246 & -34 & 248 \\
\hline 23 & 5 & 162 & 176 & 142 & -25 & 211 & 62 & 254 \\
\hline 24 & -171 & 108 & 42 & 217 & 15 & 224 & 137 & 312 \\
\hline 25 & -153 & 100 & 42 & 167 & 43 & 231 & -5 & 287 \\
\hline 26 & -94 & 133 & 26 & 218 & -59 & 230 & 4 & 188 \\
\hline 27 & -161 & 124 & -24 & 197 & -19 & 219 & 23 & 321 \\
\hline 28 & -134 & 92 & 162 & 127 & 11 & 237 & 93 & 249 \\
\hline 29 & -175 & 84 & 133 & 211 & -16 & 203 & -69 & 167 \\
\hline 30 & -93 & 149 & -17 & 192 & -16 & 195 & 72 & 220 \\
\hline 31 & -10 & 124 & 255 & 214 & -3 & 201 & 54 & 222 \\
\hline
\end{tabular}

Table 2: Mean and standard deviations of in-plane strain fields estimated from measured displacements in translation tests

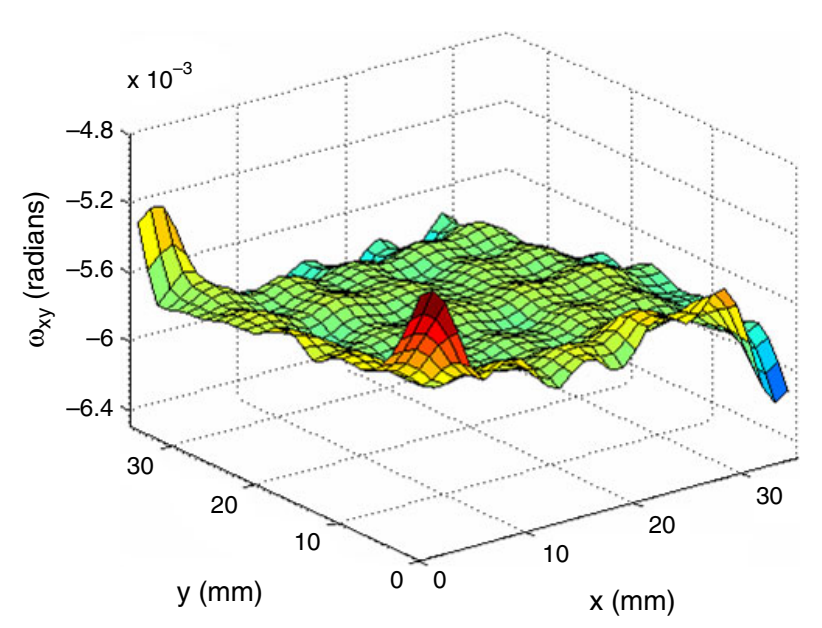

Figure 6: Estimated in-plane rotation $\omega_{X Y}$ from a pair of the images recorded by camera no. 1 value of rotation everywhere in the image except near the boundaries, particularly the corners. The deviations at the boundaries are expected because errors in derivatives of displacements (strains and rotations) get magnified near the boundaries because of the so-called edge effects. Then, the mean and standard deviations of $\omega_{X Y}$ were computed for each image (while computing these quantities for a $32 \times 32$ matrix, three rows and three columns of data points were excluded near the edge of the image because of the presence of errors at these points). Figure 7 shows the mean and standard deviations of rotations and strains from this test. It can be seen from Figure 7A,C that an applied rotation of $0.0056 \pm 0.00035 \mathrm{rad}$ is measured by all individual cameras within an error band of $\sim 0.0005 \mathrm{rad}$ ( $\sim 10 \%$ 

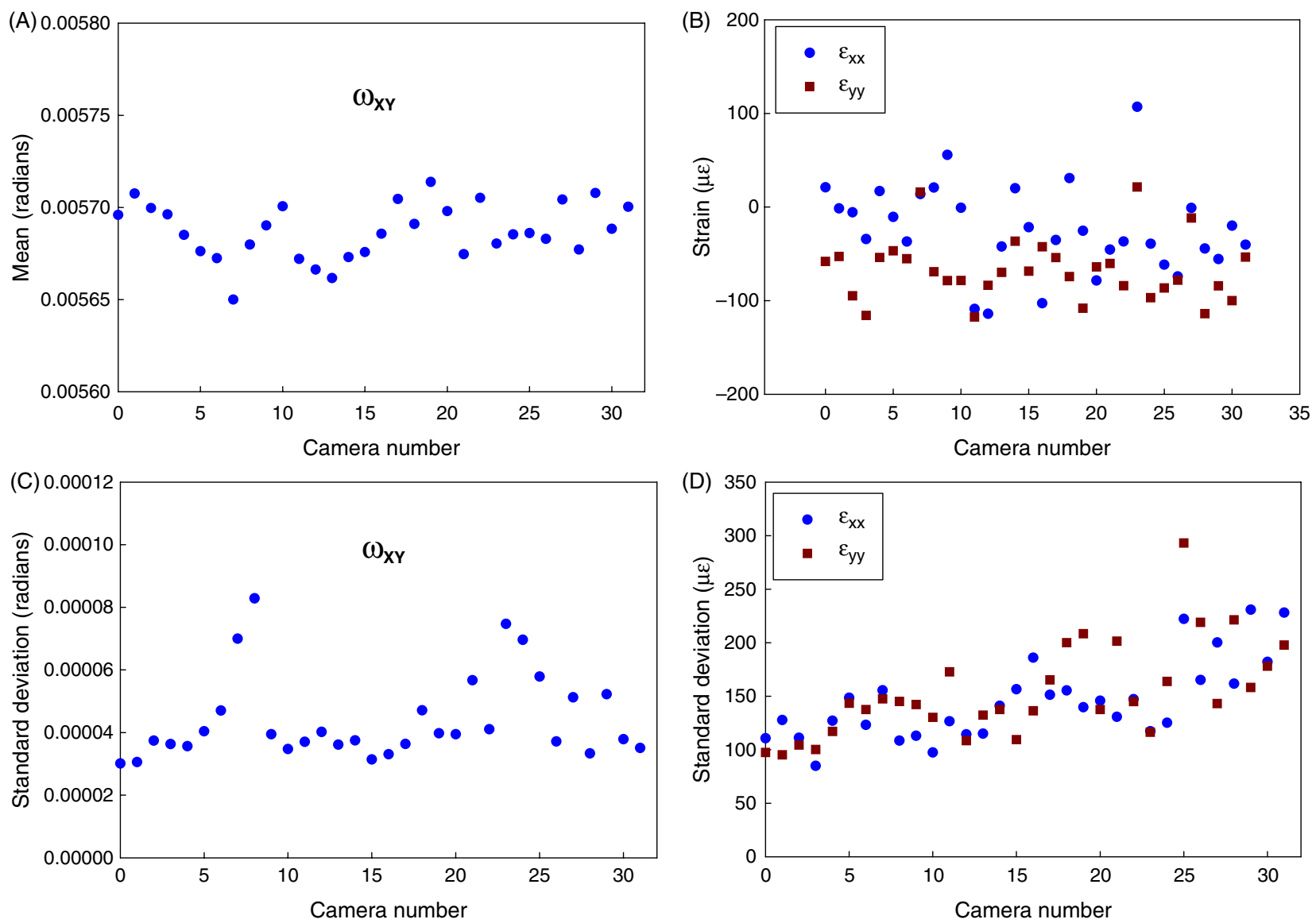

Figure 7: Results from rotation test (applied rotation $=0.0056 \pm 0.00035 \mathrm{rad}$ ). (A) Mean and (C) standard deviation of rotation field estimated from image correlation. (B) Mean (D) standard deviation of in-plane strains estimated (ideally these strains need to be zeros)

error). A rigid rotation imposed to the sample should not produce any strains. Consequently, zero strains are expected from this test. The mean values of strain fields obtained are within $120 \mu \varepsilon$ and standard deviations are up to $300 \mu \varepsilon$.

\section{Mixed-Mode Dynamic Fracture Experiment}

\section{Sample preparation}

Edge-cracked syntactic foam samples were prepared for conducting mixed-mode dynamic fracture experiments. These samples were processed by mixing 25\% (by volume) of hollow microballoons in a low-viscosity epoxy matrix. Room temperature curing [prepared by mixing a bisphenol-A resin and an amine based hardener [supplied by Buehler Corp. as 'Epo-Thin' (Buehler, Lake Bluff, IL, USA)] in the ratio $100: 38$ ] was used for sample preparation. The microballoons used in this study were commercially available hollow glass spheres (supplied by 3M Corp.) of mean diameter of $\sim 60 \mu \mathrm{m}$ and wall thickness $\sim 600 \mathrm{~nm}$. The elastic modulus and Poisson's ratio of the cured material (measured ultrasonically) were
$3.02 \pm 0.1 \mathrm{GPa}$ and $0.34 \pm 0.01$ respectively [18] Before casting the epoxy resin-hardener mixture, a sharp razor blade was inserted into the mould. After the sample was cured and removed from the mould, an edge 'crack' was left behind in the specimen [19]. Finally, the specimen was machined into a beam of height $50 \mathrm{~mm}$ with a crack of $10 \mathrm{~mm}$ length $(a / W=$ 0.2) as shown in Figure 8A. Subsequently, a random speckle pattern was created on the specimen surface by spraying with black and white paints.

\section{Experimental procedure}

As the fracture event to be captured is stress wavedominated, the total duration of recording is relatively short and hence the high-speed camera was synchronised with impact. The sequence of events in a typical experiment was as follows: the specimen was initially rested on two instrumented supports/ anvils. The camera was focused on a $31 \times 31 \mathrm{~mm}^{2}$ region of the sample in the vicinity of the crack tip (see Figure $8 \mathrm{~A}$ ). A set of 32 pictures of the stationary sample were recorded at 200000 frames per second and stored. Then an impactor was launched (velocity 


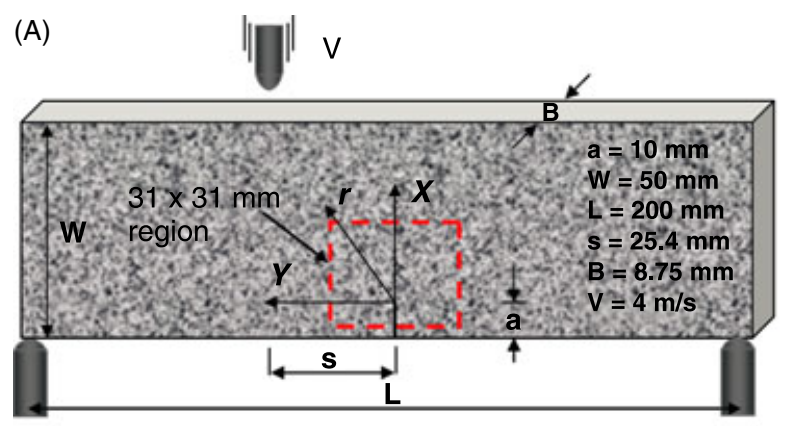

(C)
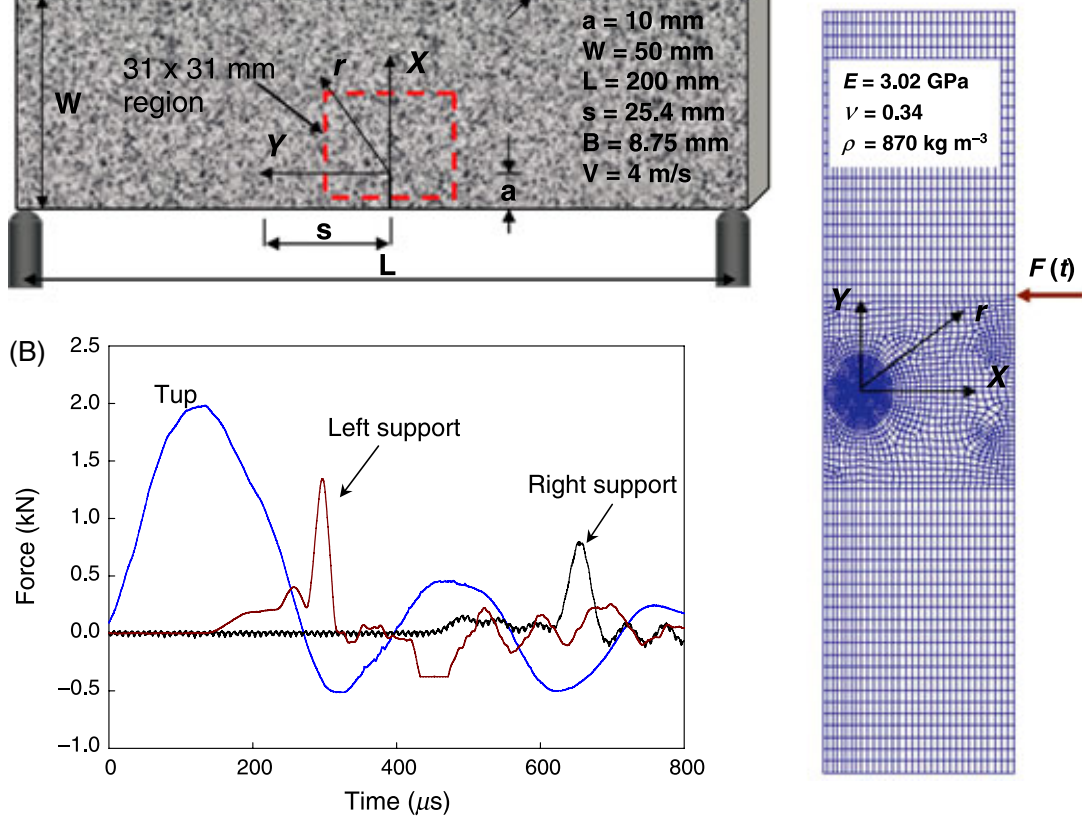

Figure 8: (A) Specimen configuration for mixed-mode dynamic fracture experiment; (B) impactor force and support reaction histories recorded by Instron-Dynatup 9250 HV drop tower and (C) finite element mesh used for elasto-dynamic simulations

of $4.0 \mathrm{~m} \mathrm{~s}^{-1}$ ) towards the sample. As soon as the impactor contacted an adhesively backed copper tape affixed to the top of the specimen, a trigger signal was generated by a pulse/delay generator and was fed into the camera. The camera then sent a separate trigger signal to a pair of high-intensity flash lamps situated symmetrically with respect to the optical axis of the camera. A time delay was pre-set in the camera to capture images $85 \mu$ s after the initial impact/contact. This time delay provided sufficient time for the high-intensity flash lamps to ramp up to their full intensity levels and provide constant intensity illumination during the recording period. As the measurable deformations around the crack tip during the first $85 \mu$ s were relatively small, there was no significant loss of information because of this delay. A total of 32 images were recorded with $5 \mu \mathrm{s}$ intervals between images, for a total duration of $160 \mu \mathrm{s}$. Once the experiment was complete, the recorded images were stored in the computer. Just before the impact occurred, the velocity of the tup was recorded [by the Instron Dynatup drop-tower system (Instron, Norwood, MA, USA)]. Tup force and support reaction histories were also recorded. These are shown in Figure 8B. In this plot, the multiple contacts between the tup and specimen can be inferred from multiple peaks. The crack initiation in this experiment occurred at about $175 \mu$ s and the crack traversed across the specimen width in the next 60-70 $\mu$ s.
Therefore, only the first peak of the impact force history is of relevance here. As the left support was closer to the impact point compared with the right, impact force record starts earlier for the former. Moreover, it should be noted that anvils register noticeable impact force after $220 \mu$ s by which time the crack propagates through half the sample width. Thus, reactions from the two anvils play no role in the fracture of the sample up to this point. Accordingly, the sample was subsequently modelled as a free-free beam in finite element simulations.

\section{Finite element simulations}

Elasto-dynamic finite element simulations of the current problem were carried out up to crack initiation under plane stress conditions. The finite element mesh used is shown in Figure 8C along with the force boundary conditions at the impact point. Experimentally determined material properties (elastic modulus $=3.1 \mathrm{GPa}$, Poisson's ratio 0.34 and mass density $=870 \mathrm{~kg} \mathrm{~m}^{-3}$ ) were used as inputs for the analysis. The numerical model was loaded using the force history recorded by the instrumented tup. [Before applying force boundary conditions, the tup force history was interpolated and smoothed for the following two reasons: (i) the time step of the tup force history measurement was larger than the one used in simulations and (ii) the force history recorded 


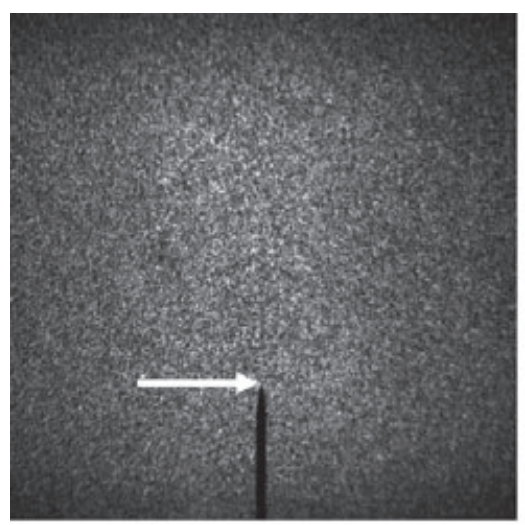

$t=170 \mu \mathrm{s}$

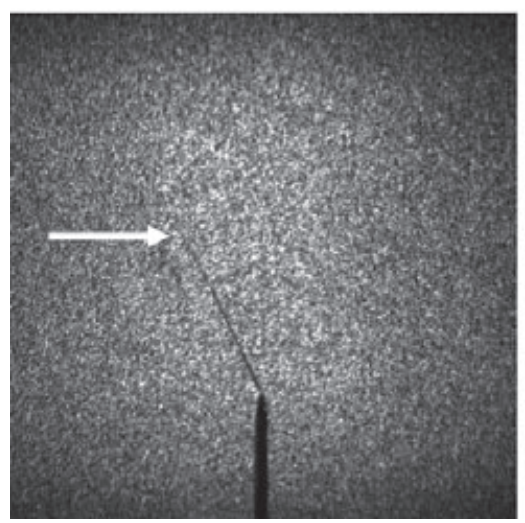

$t=215 \mu \mathrm{s}$

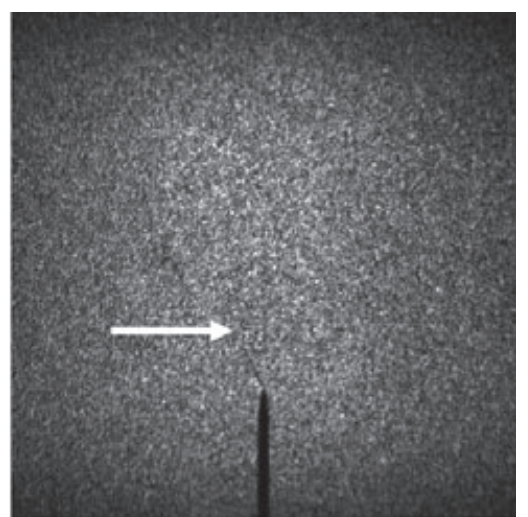

$t=190 \mu \mathrm{s}$

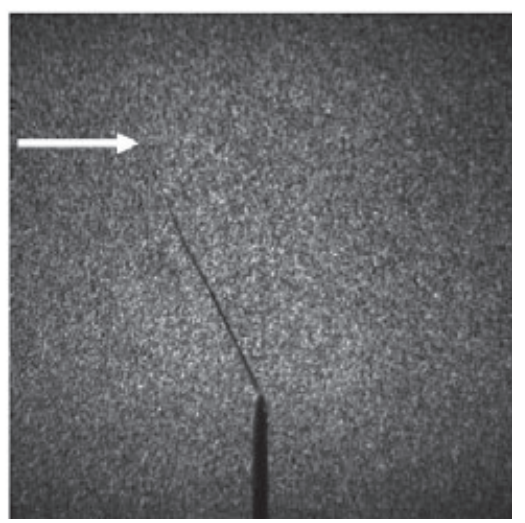

$t=240 \mu \mathrm{s}$

Figure 9: Acquired speckle images of $31 \times 31 \mathrm{~mm}^{2}$ region near a mixed-mode crack at various times instants. Current crack tip location is shown by an arrow

by the tup had experimental noise. Therefore smoothed cubic splines were fitted to the data before applying to the model.] The implicit time integration scheme of the Newmark $\beta$ method (integration parameters $\beta=0.25$ and $\gamma=0.5$ and $0.5 \%$ damping) was adopted. The details of finite element analysis can be found elsewhere [19]. The simulation results were used to obtain instantaneous SIFs up to crack initiation. The mode I and mode II SIF were calculated by regression analyses of crack-opening and sliding displacements respectively.

\section{Results}

From each experiment 64 images were available, 32 from the undeformed set and 32 from the deformed set, each having a resolution of $1000 \times 1000$ pixels. Figure 9 shows four selected speckle images from the deformed set of 32 images. The time instant at which the images were recorded after impact is indicated below each image and the instantaneous crack tip is denoted by an arrow. The crack length history is plotted in Figure 10. The crack initiates at about $175 \mu$ s. Upon initiation, it rapidly accelerates and

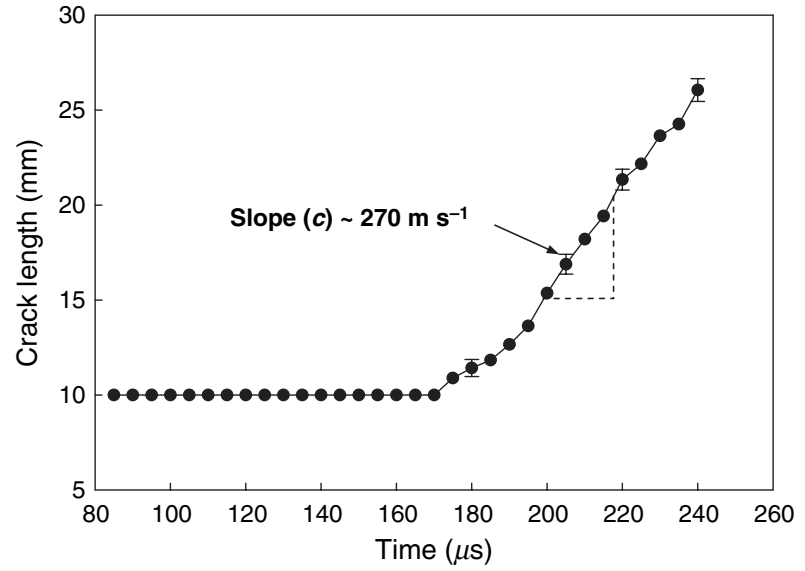

Figure 10: Crack growth history in syntactic foam sample under mixed-mode dynamic loading

subsequently reaches a nearly steady velocity of $\sim 270 \mathrm{~m} \mathrm{~s}^{-1}$. The magnification used in this experiment was such that the size of one pixel was equivalent to $31 \mu \mathrm{m}$ on the specimen surface. A sub-image size of $26 \times 26$ pixels was chosen for image correlation purpose. The in-plane displacements were estimated for all the 32 image-pairs and were resolved to an accuracy of 2-6\% of a pixel (or 0.6-1.8 $\mu \mathrm{m}$ ). The crack-opening displacement, $v$, and sliding displace- 

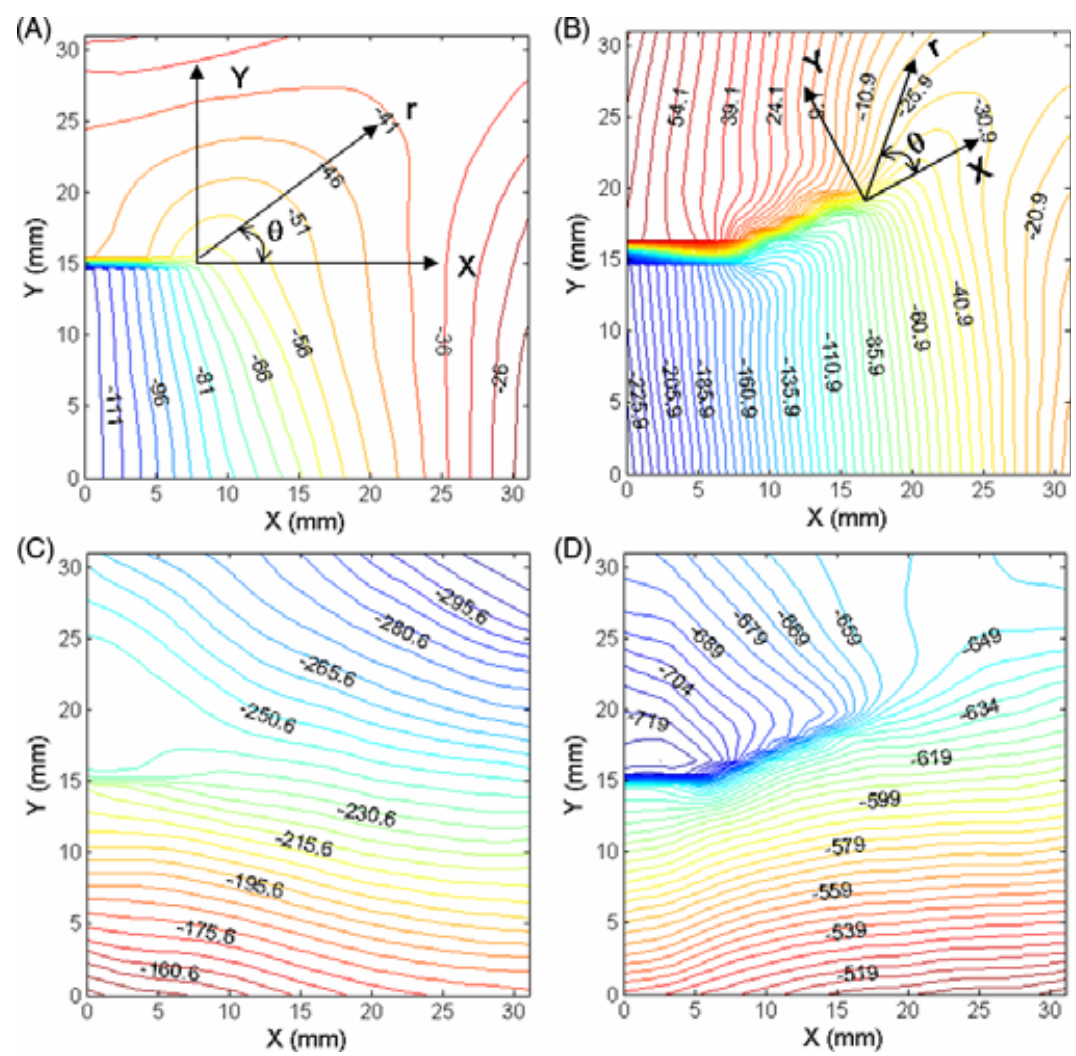

Figure I I: Crack-opening and sliding displacements (in $\mu \mathrm{m}$ ) for pre- and post-crack initiation time instants: (A) $v$-displacement $\left(u_{Y}\right)$ and $(\mathrm{C}) u$-displacement $\left(u_{X}\right)$ before crack initiation $($ at $t=150 \mu \mathrm{s}) ;(\mathrm{B}) v$-displacement $\left(u_{Y}\right)$ and $(\mathrm{D}) u$-displacement $\left(u_{X}\right)$ after crack initiation $(t=220 \mu \mathrm{s})$. Crack initiation time $\sim 175 \mu$ s. (A large rigid body displacement can be seen in (C) and (D); $u_{X}$ and $u_{Y}$ denote displacements relative to the reference grid.)

ment, $u$, for two sample images (one before crack initiation and the other after) are shown in Figure 11. Figure $11 \mathrm{~A}, \mathrm{C}$ shows $v$ - and $u$ - displacements at $150 \mu \mathrm{s}$ and Figure 11B,D shows the same displacement components, respectively, at $220 \mu$ s after impact. A significant amount of rigid-body displacement can be seen in the $u$-field in the direction of impact (Figure 11C,D).

\section{Extraction of SIFs}

Both crack-opening and sliding displacement fields were used to extract dynamic SIFs in the current study. The asymptotic expressions for a dynamically loaded stationary crack are given by [20],

$$
\begin{aligned}
u_{x}= & \sum_{n=1}^{N} \frac{\left(K_{\mathrm{I}}\right)_{n}}{2 \mu} \frac{r^{n / 2}}{\sqrt{2 \pi}}\left\{\kappa \cos \frac{n}{2} \theta-\frac{n}{2} \cos \left(\frac{n}{2}-2\right) \theta\right. \\
& \left.+\left\{\frac{n}{2}+(-1)^{n}\right\} \cos \frac{n}{2} \theta\right\} \\
& +\sum_{n=1}^{N} \frac{\left(K_{\mathrm{II}}\right)_{n}}{2 \mu} \frac{r^{n / 2}}{\sqrt{2 \pi}}\left\{\kappa \sin \frac{n}{2} \theta-\frac{n}{2} \sin \left(\frac{n}{2}-2\right) \theta\right. \\
& \left.+\left\{\frac{n}{2}-(-1)^{n}\right\} \sin \frac{n}{2} \theta\right\},
\end{aligned}
$$

$$
\begin{aligned}
u_{y}= & \sum_{n=1}^{N} \frac{\left(K_{\mathrm{I}}\right)_{n}}{2 \mu} \frac{r^{n / 2}}{\sqrt{2 \pi}}\left\{\kappa \sin \frac{n}{2} \theta+\frac{n}{2} \sin \left(\frac{n}{2}-2\right) \theta\right. \\
& \left.-\left\{\frac{n}{2}+(-1)^{n}\right\} \sin \frac{n}{2} \theta\right\} \\
& +\sum_{n=1}^{N} \frac{\left(K_{\mathrm{II}}\right)_{n}}{2 \mu} \frac{r^{n / 2}}{\sqrt{2 \pi}}\left\{-\kappa \cos \frac{n}{2} \theta-\frac{n}{2} \cos \left(\frac{n}{2}-2\right) \theta\right. \\
& \left.+\left\{\frac{n}{2}-(-1)^{n}\right\} \cos \frac{n}{2} \theta\right\},
\end{aligned}
$$

In the above equations, $u_{x}(\equiv u)$ and $u_{y}(\equiv v)$ are crack-sliding and opening displacements, $(r, \theta)$ are crack-tip polar coordinates, $\kappa$ is $(3-v) /(1+v)$ for plane stress where $\mu$ and $v$ are shear modulus and Poisson's ratio respectively. The coefficients $\left(K_{\mathrm{I}}\right)_{n}$ and $\left(K_{\mathrm{II}}\right)_{n}$ of the leading terms $(n=1)$ are the mode I and mode II dynamic SIF respectively. Equations (2) and (3) implicitly assume that inertial effects enter the coefficients while retaining the functional form of the quasi-static crack-tip equations. However, once the crack is initiated, the asymptotic expressions for sliding and opening displacements for a steadily propagating crack (assuming transient effects are negligible) are used [21]: 


$$
\begin{aligned}
u_{x}= & \sum_{n=1}^{N} \frac{\left(K_{\mathrm{I}}\right)_{n} B_{\mathrm{I}}(C)}{2 \mu} \sqrt{\frac{2}{\pi}}(n+1) \\
& \left\{r_{1}^{n / 2} \cos \frac{n}{2} \theta_{1}-h(n) r_{2}^{n / 2} \cos \frac{n}{2} \theta_{2}\right\} \\
+ & \sum_{n=1}^{N} \frac{\left(K_{\mathrm{II}}\right)_{n} B_{\mathrm{II}}(C)}{2 \mu} \sqrt{\frac{2}{\pi}}(n+1) \\
& \left\{r_{1}^{n / 2} \sin \frac{n}{2} \theta_{1}-h(\bar{n}) r_{2}^{n / 2} \sin \frac{n}{2} \theta_{2}\right\}, \\
u_{y}= & \sum_{n=1}^{N} \frac{\left(K_{\mathrm{I}}\right)_{n} B_{\mathrm{I}}(C)}{2 \mu} \sqrt{\frac{2}{\pi}}(n+1) \\
& \left\{-\beta_{1} r_{1}^{n / 2} \sin \frac{n}{2} \theta_{1}+\frac{h(n)}{\beta_{2}} r_{2}^{n / 2} \sin \frac{n}{2} \theta_{2}\right\} \\
+ & \sum_{n=1}^{N} \frac{\left(K_{\mathrm{II}}\right)_{n} B_{\mathrm{II}}(C)}{2 \mu} \sqrt{\frac{2}{\pi}}(n+1) \\
& \left\{\beta_{1} r_{1}^{n / 2} \cos \frac{n}{2} \theta_{1}+\frac{h(\bar{n})}{\beta_{2}} r_{2}^{n / 2} \cos \frac{n}{2} \theta_{2}\right\},
\end{aligned}
$$

where

$$
\begin{aligned}
& r_{m}=\sqrt{X^{2}+\beta_{m}^{2} Y^{2}}, \quad \theta_{m}=\tan ^{-1}\left(\frac{\beta_{m} Y}{X}\right) m=1,2 \\
& \beta_{1}=\sqrt{1-\left(\frac{c}{C_{\mathrm{L}}}\right)^{2}}, \beta_{2}=\sqrt{1-\left(\frac{c}{C_{\mathrm{S}}}\right)^{2}} \\
& C_{\mathrm{L}}=\sqrt{\frac{(\kappa+1) \mu}{(\kappa-1) \rho}}, C_{\mathrm{S}}=\sqrt{\frac{\mu}{\rho}}, \kappa=\frac{3-v}{1+v} \text { for plane stress } \\
& h(n)=\left\{\begin{array}{ll}
\frac{2 \beta_{1} \beta_{2}}{1+\beta_{2}^{2}} & \text { for odd } n \\
\frac{1+\beta_{2}^{2}}{2} & \text { for even } n
\end{array} \text { and } h(\bar{n})=h(n+1)\right. \\
& B_{\mathrm{I}}(c)=\frac{\left(1+\beta_{2}^{2}\right)}{D}, B_{\mathrm{II}}(c)=\frac{2 \beta_{2}}{D}, D=4 \beta_{1} \beta_{2}-\left(1+\beta_{2}^{2}\right)^{2} .
\end{aligned}
$$

Here $(x, y)$ and $(r, \theta)$ are the Cartesian and polar coordinates instantaneously aligned with the current crack tip, respectively (see, Figure 11B), $c$ is crack speed, $C_{\mathrm{L}}$ and $C_{\mathrm{S}}$ are dilatational and shear wave speeds in the material, and $\mu$ and $v$ are shear modulus and Poisson's ratio respectively. Again, coefficients $\left(K_{\mathrm{I}}\right)_{n}$ and $\left(K_{\mathrm{II}}\right)_{n}$ of the leading terms are the mode I and mode II dynamic SIFs respectively.

For a mode I problem, $u_{y}(\equiv v)$ is the dominant in-plane displacement and is generally used for extracting mode I SIF history. However, in a mixedmode problem, both $u_{x}$ and $u_{y}$ displacements are present. The crack-opening displacements $\left(u_{y}\right)$ can be viewed as those with mode I-rich information whereas crack sliding displacements $\left(u_{x}\right)$ with mode II-rich information. Thus, $u_{y}$ can be used to extract $K_{\mathrm{I}}$ and $u_{x}$ to extract $K_{\mathrm{II}}$ accurately. Alternatively, either radial $\left(u_{r}\right)$ or tangential $\left(u_{\theta}\right)$ displacements (computed by transforming $u_{x}$ and $u_{y}$ data) can be used to extract both $K_{\mathrm{I}}$ and $K_{\mathrm{II}}$ more accurately instead of using $u_{x}$ and $u_{y}$ individually. This has been vividly demon- strated by Yoneyama et al. [22] in a recent article. Accordingly, in this study both radial and tangential displacement components $u_{r}$ and $u_{\theta}$ were used to extract both $K_{\mathrm{I}}$ and $K_{\mathrm{II}}$ histories.

For extracting SIF from displacement data, the current crack-tip location was identified and the Cartesian and polar coordinate systems $(x-y$ and $r-\theta)$ were established. A set of data points (usually 100 to 120) were collected in the region around the crack tip in the domain $0.3<r / B<1.6$ and $-135^{\circ}<\theta<135^{\circ}$, $(B$ is sample thickness). At each data point, $u_{x}$ and $u_{y}$ displacement values as well as the location of these points were stored. Then $u_{x}$ and $u_{y}$ were transformed into $u_{r}$ and $u_{\theta}$ by

$\left\{\begin{array}{l}u_{r} \\ u_{\theta}\end{array}\right\}=\left[\begin{array}{cc}\cos \theta & \sin \theta \\ -\sin \theta & \cos \theta\end{array}\right]\left\{\begin{array}{l}u_{x} \\ u_{y}\end{array}\right\}$.

The asymptotic expressions for $u_{r}$ and $u_{\theta}$ were also transformed from $u_{x}$ and $u_{y}$ as follows:

$$
\begin{aligned}
u_{r k}= & \left\{\sum_{n=1}^{N}\left(K_{\mathrm{I}}\right)_{n} f_{\mathrm{I} n}\left(r_{k}, \theta_{k}\right)+\sum_{n=1}^{N}\left(K_{\mathrm{II}}\right)_{n} f_{\mathrm{II} n}\left(r_{k}, \theta_{k}\right)\right\} \cos \theta_{k} \\
& +\left\{\sum_{n=1}^{N}\left(K_{\mathrm{I}}\right)_{n} g_{\mathrm{II} n}\left(r_{k}, \theta_{k}\right)+\sum_{n=1}^{N}\left(K_{\mathrm{II}}\right)_{n} g_{\mathrm{III} n}\left(r_{k}, \theta_{k}\right)\right\} \sin \theta_{k}, \\
& +T_{x} \cos \theta_{k}+T_{y} \sin \theta_{k}, \\
u_{\theta k}= & -\left\{\sum_{n=1}^{N}\left(K_{\mathrm{I}}\right)_{n} f_{\mathrm{I} n}\left(r_{k}, \theta_{k}\right)-\sum_{n=1}^{N}\left(K_{\mathrm{II}}\right)_{n} f_{\mathrm{II} n}\left(r_{k}, \theta_{k}\right)\right\} \sin \theta_{k} \\
& +\left\{\sum_{n=1}^{N}\left(K_{\mathrm{I}}\right)_{n} g_{\mathrm{II} n}\left(r_{k}, \theta_{k}\right)+\sum_{n=1}^{N}\left(K_{\mathrm{II}}\right)_{n} g_{\mathrm{III} n}\left(r_{k}, \theta_{k}\right)\right\} \cos \theta_{k}, \\
& -T_{x} \sin \theta_{k}+T_{y} \cos \theta_{k},
\end{aligned}
$$

where $f_{\text {In }}, f_{\text {II } n}, g_{\text {In } n}$ and $g_{\text {IIn } n}$ are angular functions from Equations (2) and (3) or (4) and (5). In Equations (8) and (9) subscripts ' $n$ ' and ' $k$ ' are indices used to represent the number of terms in the expansion (usually three were found sufficient in this study) and the number of data points at which displacements were collected. Further, $T_{x}$ and $T_{y}$ denote rigid body translation in the $x$ - and $y$-directions respectively. An over-deterministic least-squares analysis [23] of the data set was carried out in order to estimate $K_{\mathrm{I}}$ and $K_{\mathrm{II}}$. This was repeated for all the 32 image pairs to generate SIF histories.

Figure 12(A) and (B) shows SIF histories extracted from displacements $u_{r}$ and $u_{\theta}$ respectively. The time at which crack initiation occurs is indicated by a dotted line in both figures. Three different plots (solid symbols) are presented in Figure 12A,B for both $K_{\mathrm{I}}$ and $K_{\mathrm{II}}$ obtained by varying the number of terms of the asymptotic series. A good convergence is readily 

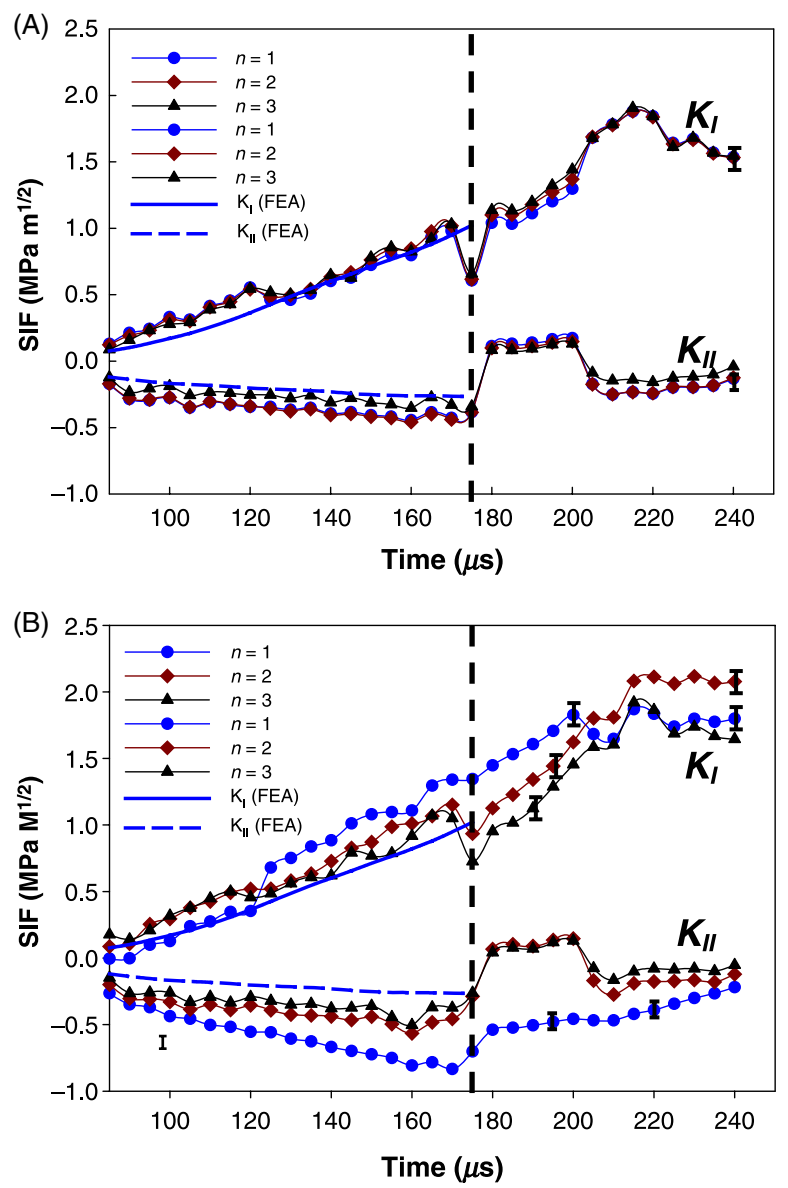

Figure 12: Stress intensity factors extracted from displacement fields: SIF histories from (A) $u_{r}$ displacement and (B) from $u_{\theta}$ displacement. SIF history obtained from finite element simulation up to crack initiation is also shown. The broken line corresponds to crack initiation time

evident from Figure 12A when the number of terms $(n=1,2,3)$ was used in the expansion for $u_{r}$. Interestingly, the differences between the three plots are rather small. This suggests that higher order terms ( $n=2$ and 3 ) improve results obtained from using just the $n=1$ term only marginally in this case. In contrast, SIF histories evaluated for $n=1,2,3$ from $u_{\theta}$ (Figure 12B) show differences to a larger extent. That is, here the plots are noticeably separated from each other, implying that the $u_{\theta}$ field is influenced by the higher order terms more strongly. This type of behaviour is expected because the higher order terms influence different field quantities differently and has been exploited advantageously in strain gauge-based methods as well as other optical methods used for extracting SIFs [19, 24-26].

Both mode I and mode II SIFs increase monotonically up to crack initiation (at $175 \mu \mathrm{s}$ ). Following crack initiation there is a noticeable dip in both $K_{\mathrm{I}}$ and $K_{\mathrm{II}}$ because of elastic unloading near the crack tip. In the pre-initiation regime, the $K_{\text {II }}$ values are negative, consistent with the loading configuration

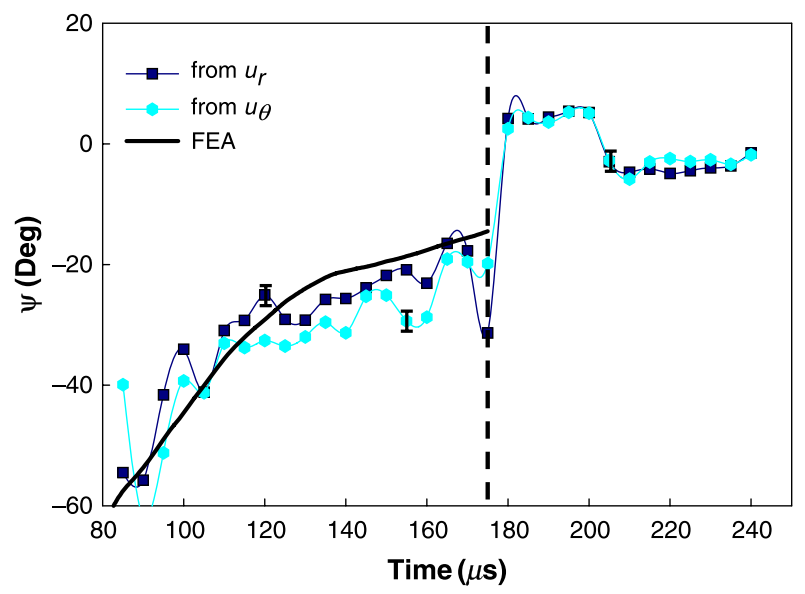

Figure 13: The mode-mixity $\psi\left[=\tan ^{-1}\left(K_{\mathrm{II}} / K_{\mathrm{I}}\right)\right]$ deduced from experimentally obtained SIFs as with $N=3$ in Equations (8) and (9). The $\psi$ obtained from finite element simulations up to crack initiation is also shown. The broken line corresponds to crack initiation time

on hand and the crack kink direction following initiation. After initiation at $K_{\mathrm{I}} \sim 1.0 \mathrm{MPam}^{1 / 2}, K_{\mathrm{I}}$ continues to increase until it reaches a value of $\sim 1.8 \mathrm{MPam}^{1 / 2}$ beyond which it shows a decreasing trend, whereas the mode II SIF, $K_{\mathrm{II}}$, remains close to zero in the post-initiation regime. The relative amount of in-plane shear stress to normal stress amplification near the crack can be quantified by mode mixity $\psi=\tan ^{-1}\left(K_{\mathrm{II}} / K_{\mathrm{I}}\right)$. The $\psi$-history is plotted in Figure 13. A large negative value for $\psi$ can be seen at the initial stages indicating the presence of a significant negative in-plane shear component at the crack tip. Just before crack initiation, however, $\psi$ approaches zero, suggesting that crack initiation and propagation occur under dominant mode I conditions $\left(K_{\mathrm{II}} \rightarrow 0\right)$. In each of the plots shown in Figure 13 , SIF and mode-mixity histories evaluated from experiments show good agreement with those from finite element computations (solid line) up to crack initiation.

\section{Estimation ${ }^{2}$ of strains}

The displacements were smoothed by the methodology explained in Ref. [12]. Smoothing was based on the noise level in the displacement data quantified $a$ priori. The following approach was adopted to estimate the noise level in the displacement data. A random speckle pattern was imaged twice with the high-speed camera system at the same framing rate (200,000 frames per second) as the one used in frac-

\footnotetext{
${ }^{2}$ As strains are obtained in this study by numerically differentiating measured displacements, the term 'estimation' is used instead of 'measured'.
} 
ture experiments. The full-field $u$ - and $v$-displacements between these two images were estimated. Ideally, one would expect both $u$ - and $v$-displacements to be zero. The extracted $u$-displacement in this study oscillated about zero and had a standard deviation $(\sigma)=0.039$ pixel and variance $=0.0019$ pixel $^{2}$. This value was accepted as the noise variance and was used while performing smoothing operation. Once the displacements were smoothed, Lagrangian strains (relative to the reference coordinates $X$ and $Y$ ) were obtained by differentiating displacements as follows:

$$
\begin{aligned}
& \varepsilon_{x X}=\frac{\partial u}{\partial X}+\frac{1}{2}\left\{\left(\frac{\partial u}{\partial X}\right)^{2}+\left(\frac{\partial v}{\partial X}\right)^{2}\right\}, \\
& \varepsilon_{y y}=\frac{\partial v}{\partial Y}+\frac{1}{2}\left\{\left(\frac{\partial u}{\partial Y}\right)^{2}+\left(\frac{\partial v}{\partial Y}\right)^{2}\right\}, \\
& \varepsilon_{x y}=\frac{1}{2}\left\{\frac{\partial u}{\partial Y}+\frac{\partial v}{\partial X}\right\}+\frac{1}{2}\left\{\frac{\partial u}{\partial X} \frac{\partial u}{\partial Y}+\frac{\partial v}{\partial X} \frac{\partial v}{\partial Y}\right\} .
\end{aligned}
$$
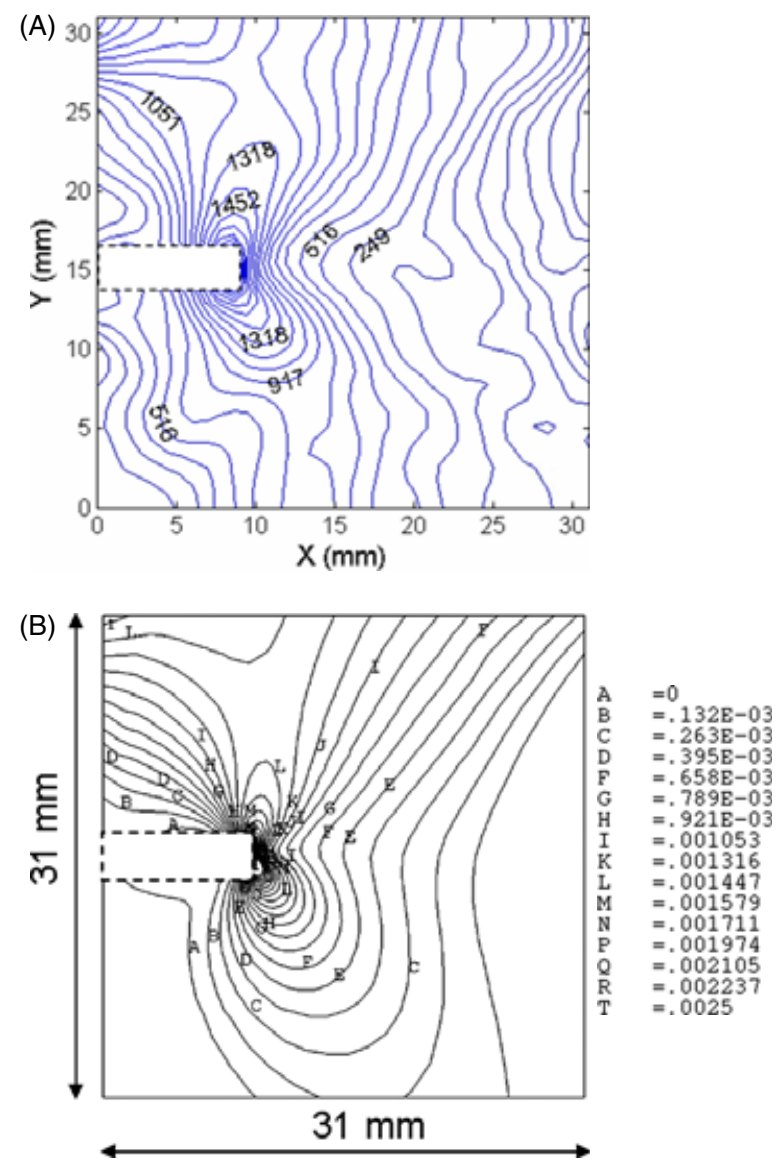

Figure 14: Crack-tip normal strains (in micro strains) at a region of $31 \times 31 \mathrm{~mm}$ around the crack tip before crack initiation at $t=150 \mu \mathrm{s}$; (A) from experiment, (B) from finite element analysis
(It should be noted that the higher order terms did not contribute significantly to strains in this study.) The crack tip normal strains obtained using this methodology are shown in Figure 14A at a time instant $150 \mu$ s after impact. The normal strain contours from finite element results at $150 \mu$ s are also shown in Figure 14B. To facilitate a direct comparison, an increment of $\sim 132 \mu \varepsilon$ was chosen between each contour level in both these plots. The concentration of $\varepsilon_{y y}$ around the mixed-mode crack tip can be readily seen in these figures. Besides quantitative agreement, a qualitative similarity between the strain contours obtained from experiments and numerical simulations close to the crack tip in Figure 14A,B can be readily seen. In the far field where the strains are below discernible levels, the agreement is expectedly modest.

\section{Conclusions}

The image correlation technique combined with high-speed digital photography was successfully developed to study mixed-mode dynamic fracture of syntactic foams under stress-wave loading conditions. A three-step approach developed by the authors was adopted to estimate deformations and strains from digitised speckle images. The entire crack-tip deformation history from the time of impact to complete specimen fracture was mapped. Over-deterministic least-squares analysis of radial and tangential displacement fields was carried out for extracting mixed-mode SIFs. The following are among the major outcomes of the current study:

- Image intensity variability test, translation test and rotation test performed for benchmarking the imaging system show that the random intensity variation in the range 0-8 grey levels exists at a pixel for the CCD-based camera system used and the applied displacements can be faithfully reproduced (2-6\% of a pixel) within acceptable experimental errors.

- A rotating mirror-type camera system with multiple optical relays can be used for performing 2D image correlation to study transient events dominated by stress waves. This requires correlation of two images captured by the same CCD sensor before and after deformation to circumvent distortions between different CCD sensors and optical relay circuits.

- The SIF histories and mode-mixities obtained from experiments are in good agreement with the those from finite element computations up to crack initiation. The mixed-mode crack initiation 
and growth in syntactic foam occur under predominantly mode I conditions and is consistent with a macroscopically homogeneous and isotropic material behaviour.

- The radial displacement field is relatively less susceptible to higher order effects when compared with the tangential displacements for the mixedmode crack experiment considered.

\section{ACKNOWLEDGEMENT}

The authors would like to thank the U.S. Army Research Office for supporting this research through grants W911NF-04-10257 and DAAD19-02-10126 (DURIP).

\section{REFERENCES}

1. de Graaf, J. G. A. (1964) Investigation of brittle fracture in steel by means of ultra high speed photography. Appl. Opt. 3, 1223-1229.

2. Dally, J. W. (1979) Dynamic photo-elastic studies of fracture. Exp. Mech. 19, 349-361.

3. Parameswaran, V. and Shukla, A. (1998) Dynamic fracture of a functionally gradient material having discrete property variation. J. Mater. Sci. 33, 3303-3311.

4. Tippur, H. V., Krishnaswamy, S. and Rosakis, A. J. (1991) A coherent gradient sensor for crack tip measurements: analysis and experimental results. Int. J. Fracture 48, 193204.

5. Rousseau,C. E. Tippur, H. V. (2001) Dynamic fracture of compositionally graded materials with cracks along the elastic gradient experiments and analysis. Mech. Mater. 33, 403-421.

6. Kirugulige, M. S., Kitey, R. and Tippur, H. V. (2004) Dynamic fracture behavior of model sandwich structures with functionally graded core: a feasibility study. Composites Sci. Technol. 65, 1052-1068.

7. Kirugulige, M. S. and Tippur, H. V. (2006) Mixed mode dynamic crack growth in functionally graded glass filled epoxy. Exp. Mech. 46, 269-281.

8. Guo, Z. K. and Kobayashi, A. S. (1995) Dynamic mixed mode fracture of concrete. Int. J. Solids Struct. 32, 25912607.

9. Hild, F. and Roux, S. (2006) Digital image correlation: from displacement measurement to identification of elastic properties - a review. Strain 42, 69-80.

10. Abanto-Bueno, J. and Lambros, J. (2006) An experimental study of mixed-mode crack initiation and growth in functionally graded materials. Exp. Mech. 46, 179-196.

11. The MathWorks, Inc. (2006) Matlab 7.0 (available at: http://www.mathworks.com).
12. Kirugulige, M. S., Tippur, H. V. and Denney, T. S. (2007) Measurement of transient deformations using digital image correlation method and high-speed photography: application to dynamic fracture. Appl. Opt. 46, 5083-5096.

13. Kirugulige, M., Tippur, H. and Denney, T. (2007) Investigation of mixed-mode dynamic fracture in syntactic foams using digital image correlation method and highspeed photography. Proc. 2007 SEM Annu. Conf. Exposition Exp. Appl. Mech. 4-7 June, Springfield, MA, USA.

14. Chen, D. J., Chiang, F. P., Tan, Y. S. and Don, H. S. (1993) Digital speckle-displacement measurement using a complex spectrum method. Appl. Opt. 32, 1839-1849.

15. Sutton M. A., Walters, W. J., Peters, W. H., Ranson, W. F. and McNeil, S. R. (1983) Determination of displacements using an improved digital image correlation method. Image Vision Comput. 1, 133-139.

16. Gonzalez, R. C., Woods, R. E. and Eddins, S. L. (2004) Digital Image Processing using MATLAB, 1st edn. Prentice Hall, Upper Saddle River, NJ.

17. Tiwari, V., Sutton, M., McNeill, K. O., Fourney, W. and Bretall, D. (2007) On studying improvised explosive devices (IEDs) using digital image correlations. Proc. 2007 SEM Annu. Conf. Exposition Exp. Appl. Mech., Springfield, MA, USA.

18. Butcher, R. J., Rousseau, C.-E., and Tippur, H. V. (1998) A functionally graded particulate composite: preparation, measurements and failure analysis. Acta Mater. 47, 259268.

19. Maleski, M. J., Kirugulige, M. S. and Tippur, H. V. (2004) A method for measuring mode-I crack tip constraint under static and dynamic loading conditions. Exp. Mech. 44, 522-532.

20. Westergaard, H. M. (1939) Bearing pressure and cracks. ASME J. Appl. Mech. 6, 49-53.

21. Nishioka, T. and Atluri, S. N. (1983) Path independent integrals, energy release rates, and general solutions of near-tip fields in mixed-mode dynamic fracture mechanics. Eng. Fracture Mech. 18, 1-22.

22. Yoneyama, S., Morimoto, Y. and Takashi, M. (2006) Automatic evaluation of mixed-mode stress intensity factors utilizing digital image correlation. Strain 42, 21-29.

23. Dally, J. W. and Riley, W. F. (2005) Experimental Stress Analysis, 4th edn. College House Enterprises, LLC, Knoxville, TN.

24. Khanna, S. K. and Shukla, A. (1995) On the use of strain gages in dynamic fracture mechanics. Eng. Fracture Mech. 51, 933-948.

25. Dally, J. W. and Sanford, R. J. (1987) Strain-gage methods for measuring the opening-mode stress intensity factor, K. Exp. Mech. 49, 381-388.

26. Sanford, R. J. (2003) Principles of Fracture Mechanics. Prentice Hall, Upper Saddle River, NJ. 